\title{
Geometrical versions of improved Berezin-Li-Yau inequalities
}

\author{
Leander Geisinger, Ari Laptev and Timo Weidl ${ }^{1}$
}

\begin{abstract}
We study the eigenvalues of the Dirichlet Laplace operator on an arbitrary bounded, open set in $\mathbb{R}^{d}, d \geq 2$. In particular, we derive upper bounds on Riesz means of order $\sigma \geq 3 / 2$, that improve the sharp Berezin inequality by a negative second term. This remainder term depends on geometric properties of the boundary of the set and reflects the correct order of growth in the semi-classical limit.

Under certain geometric conditions these results imply new lower bounds on individual eigenvalues, which improve the $\mathrm{Li}-$ Yau inequality.
\end{abstract}

Mathematics Subject Classification (2010). Primary 35P15; Secondary 47A75.

Keywords. Dirichlet-Laplace operator, semi-classical estimates, Berezin-Li-Yau inequality.

\section{Introduction}

Let $\Omega \subset \mathbb{R}^{d}$ be an open set and let $-\Delta$ denote the Dirichlet Laplace operator on $L^{2}(\Omega)$, defined as a self-adjoint operator with form domain $H_{0}^{1}(\Omega)$. We assume that the volume of $\Omega$, denoted by $|\Omega|$, is finite. Then the embedding $H_{0}^{1}(\Omega) \hookrightarrow L^{2}(\Omega)$ is compact and the spectrum of $-\Delta$ is discrete: it consists of positive eigenvalues

$$
0<\lambda_{1} \leq \lambda_{2} \leq \lambda_{3} \leq \cdots
$$

accumulating at infinity only.

Here we are interested in upper bounds on the Riesz means

$$
\sum_{k}\left(\Lambda-\lambda_{k}\right)_{+}^{\sigma}=\operatorname{Tr}(-\Delta-\Lambda)_{-}^{\sigma}, \quad \sigma \geq 0,
$$

where we use the notation $x_{ \pm}=(|x| \pm x) / 2$. In 1972 Berezin proved that convex eigenvalue means are bounded uniformly by the corresponding phase-space volume, see [2]: for any open set $\Omega \subset \mathbb{R}^{d}, \sigma \geq 1$, and all $\Lambda>0$

$$
\operatorname{Tr}(-\Delta-\Lambda)_{-}^{\sigma} \leq \frac{1}{(2 \pi)^{d}} \iint_{\Omega \times \mathbb{R}^{d}}\left(|p|^{2}-\Lambda\right)_{-}^{\sigma} d p d x=L_{\sigma, d}^{\mathrm{cl}}|\Omega| \Lambda^{\sigma+d / 2},
$$

\footnotetext{
${ }^{1}$ Financial support from the German Academic Exchange Service (DAAD) and the British Council (ARC Project 50022370) is gratefully acknowledged. The first author wants to thank for the hospitality at Imperial College London.
} 
see also [27], where the problem is treated from a different point of view. Here $L_{\sigma, d}^{\mathrm{cl}}$ denotes the so-called Lieb-Thirring constant

$$
L_{\sigma, d}^{\mathrm{cl}}=\frac{\Gamma(\sigma+1)}{(4 \pi)^{d / 2} \Gamma(\sigma+1+d / 2)} .
$$

The Berezin inequality (1) captures, in particular, the well-known asymptotic limit that goes back to Hermann Weyl [34]: for $\Omega \subset \mathbb{R}^{d}$ and $\sigma \geq 0$ the asymptotic identity

$$
\operatorname{Tr}(-\Delta-\Lambda)_{-}^{\sigma}=L_{\sigma, d}^{\mathrm{cl}}|\Omega| \Lambda^{\sigma+d / 2}+o\left(\Lambda^{\sigma+d / 2}\right)
$$

holds true as $\Lambda \rightarrow \infty$. From this follows that the Berezin inequality is sharp, in the sense that the constant in (1) cannot be improved. However, Hermann Weyl's work stimulated further analysis of the asymptotic formula and (2) was gradually improved by studying the second term, see [4], [19], [21], [28], [31], [22], and references within. The precise second term was found by Ivrii [21]: under appropriate conditions on the set $\Omega$ and its boundary $\partial \Omega$ the relation

$$
\begin{aligned}
\operatorname{Tr}(-\Delta-\Lambda)_{-}^{\sigma}= & L_{\sigma, d}^{\mathrm{cl}}|\Omega| \Lambda^{\sigma+d / 2}-\frac{1}{4} L_{\sigma, d-1}^{\mathrm{cl}}|\partial \Omega| \Lambda^{\sigma+(d-1) / 2} \\
& +o\left(\Lambda^{\sigma+(d-1) / 2}\right)
\end{aligned}
$$

holds as $\Lambda \rightarrow \infty$. To simplify notation we write $|\Omega|$ for the volume (the $d$-dimensional Lebesgue measure) of $\Omega$, as well as $|\partial \Omega|$ for the $(d-1)$-dimensional surface area of its boundary. Since the second term of this semi-classical limit is negative, the question arises, whether the Berezin inequality (1) can be improved by a negative remainder term.

Recently, several such improvements have been found, initially for the discrete Laplace operator, see [11]. The first result for the continuous Laplace operator is due to Melas [29]. From his work follows that

$$
\operatorname{Tr}(-\Delta-\Lambda)_{-}^{\sigma} \leq L_{\sigma, d}^{\mathrm{cl}}|\Omega|\left(\Lambda-M_{d} \frac{|\Omega|}{I(\Omega)}\right)_{+}^{\sigma+d / 2}, \quad \Lambda>0, \sigma \geq 1,
$$

where $M_{d}$ is a constant depending only on the dimension and $I(\Omega)$ denotes the second moment of $\Omega$, see also [20], [36], and [37] for further generalisations. One should mention, however, that these corrections do not capture the correct order in $\Lambda$ from the second term of the asymptotics (3). This was improved in the two-dimensional case in [23], where it is shown that one can choose the order of the correction term arbitrarily close to the correct one.

In this paper we are interested in the case $\sigma \geq 3 / 2$. For these values of $\sigma$ it is known, [33], that one can strengthen the Berezin inequality for any open set $\Omega \subset \mathbb{R}^{d}$ with a negative remainder term reflecting the correct order in $\Lambda$ in comparison to the second term of (3). However, since one can increase $|\partial \Omega|$ without changing the individual eigenvalues $\lambda_{k}$ significantly, a direct analog of the first two terms of the 
asymptotics (3) cannot yield a uniform bound on the eigenvalue means. Therefore - without further conditions on $\Omega$ - any uniform improvement of (1) must invoke other geometric quantities.

In the result from [33] the remainder term involves certain projections on (d-1)-dimensional hyperplanes. In [16] a universal improvement of (1) was found, that holds for $\sigma \geq 3 / 2$ with a correction term of correct order, depending only on the volume of $\Omega$.

The proof of the aforementioned results relies on operator-valued Lieb-Thirring inequalities [26] and an inductive argument, that allows to reduce the problem to estimating traces of the one-dimensional Dirichlet Laplace operator on open intervals.

In this paper we use the same approach, but with new estimates in the onedimensional case, in order to make the dependence on the geometry more transparent. The new one-dimensional bounds involve the distance to the boundary of the interval in question and are related to Hardy-Lieb-Thirring inequalities for Schrödinger operators, see [7]. There it is shown that, for $\sigma \geq 1 / 2$ and potentials $V \in L^{\sigma+1 / 2}\left(\mathbb{R}_{+}\right)$, given on the half-line $\mathbb{R}_{+}=(0, \infty)$, the inequality

$$
\operatorname{Tr}\left(-\frac{d^{2}}{d t^{2}}-V\right)_{-}^{\sigma} \leq L_{\sigma} \int_{0}^{\infty}\left(V(t)-\frac{1}{4 t^{2}}\right)_{+}^{\sigma+1 / 2} d t
$$

holds true, with a constant $L_{\sigma}$ independent of $V$. For further developments see [10] and [13].

We start this paper by analysing the special case of the Dirichlet Laplace operator given on a finite interval $I \subset \mathbb{R}$, with the constant potential $V \equiv \Lambda$. For $\sigma \geq 1$ we establish that the estimate

$$
\operatorname{Tr}\left(-\frac{d^{2}}{d t^{2}}-\Lambda\right)_{-}^{\sigma} \leq L_{\sigma, 1}^{\mathrm{cl}} \int_{I}\left(\Lambda-\frac{1}{4 \delta(t)^{2}}\right)_{+}^{\sigma+1 / 2} d t
$$

is valid with the sharp constant $L_{\sigma, 1}^{\mathrm{cl}}$, where $\delta(t)$ denotes the distance to the boundary of $I$. This is done in Section 2.

Then we can use results from [26] and [33] to deduce bounds in higher dimensions: in Section 3 we first derive improvements of (1), which are valid for any open set $\Omega \subset \mathbb{R}^{d}, d \geq 2$. These improvements depend on the geometry of $\Omega$. In view of the asymptotic result (3) one might expect, that this geometric dependence can be expressed in terms of the boundary of $\Omega$. To see this, we adapt methods which were used in [5], [6], and [18] to derive geometric versions of Hardy's inequality. Here the result gives an improved Berezin inequality with a correction term of correct order depending on geometric properties of the boundary.

If $\Omega$ is convex and smooth this dependence can be expressed only in terms of $|\Omega|$, $|\partial \Omega|$, and the curvature of the boundary. In particular the first remainder term of the estimate is very similar to the second term of the semiclassical asymptotics (3): it shows the same order in $\Lambda$ and it depends only on the surface area of the boundary.

In Section 4 we return to the general case, where $\Omega \subset \mathbb{R}^{d}$ is not necessarily convex or smooth, and obtain lower bounds on individual eigenvalues $\lambda_{k}$. Under 
certain conditions on the geometry of $\Omega$, these results improve the estimate

$$
\lambda_{k} \geq C_{d} \frac{d}{d+2}\left(\frac{k}{|\Omega|}\right)^{2 / d},
$$

from [27], where $C_{d}$ denotes the semi-classical constant $4 \pi \Gamma(d / 2+1)^{2 / d}$.

Finally, in Section 5, we specialise to the two-dimensional case, where we can use the foregoing results and refined geometric considerations to further improve and generalise the inequalities. In particular, we avoid dependence on curvature, thus we do not require smoothness of the boundary.

The question whether such improved estimates can be generalised to $1 \leq \sigma<3 / 2$ remains open.

\section{One-dimensional considerations}

Let us consider an open interval $I \subset \mathbb{R}$ of length $l>0$. For $t \in I$ let

$$
\delta(t)=\inf \{|t-s|: s \notin I\}
$$

be the distance to the boundary. The eigenvalues of $-d^{2} / d t^{2}$ subject to Dirichlet boundary conditions at the endpoints of $I$ are given by $\lambda_{k}=k^{2} \pi^{2} / l^{2}$. Therefore the Riesz means equal

$$
\operatorname{Tr}\left(-\frac{d^{2}}{d t^{2}}-\Lambda\right)_{-}^{\sigma}=\sum_{k}\left(\Lambda-\frac{k^{2} \pi^{2}}{l^{2}}\right)_{+}^{\sigma}
$$

To find precise bounds on the Riesz means in the one-dimensional case, it suffices to analyse this sum explicitly. Our main observation is

Lemma 2.1. Let $I \subset \mathbb{R}$ be an open interval and $\sigma \geq 1$. Then the estimate

$$
\operatorname{Tr}\left(-\frac{d^{2}}{d t^{2}}-\Lambda\right)_{-}^{\sigma} \leq L_{\sigma, 1}^{\mathrm{cl}} \int_{I}\left(\Lambda-\frac{1}{4 \delta(t)^{2}}\right)_{+}^{\sigma+1 / 2} d t
$$

holds true for all $\Lambda>0$. The constant $1 / 4$ cannot be improved.

The remainder of this section deals with the proof of this estimate. First we need two rather technical results. The proof is elementary but not trivial and therefore will be given in the appendix.

Lemma 2.2. For all $A \geq 1 / \pi$

$$
\sum_{k}\left(1-\frac{k^{2}}{A^{2}}\right)_{+} \leq \frac{2}{3 \pi} \int_{1}^{\pi A}\left(1-\frac{1}{s^{2}}\right)^{3 / 2} d s
$$


Lemma 2.3. Let $I \subset \mathbb{R}$ be an open interval of length $l>0$. Then for $\sigma \geq 1$ and $c>0$

$$
L_{\sigma, 1}^{\mathrm{cl}} \int_{I}\left(\Lambda-\frac{c}{\delta(t)^{2}}\right)_{+}^{\sigma+1 / 2} d t-\sum_{k}\left(\Lambda-\frac{\pi^{2} k^{2}}{l^{2}}\right)_{+}^{\sigma}=\left(\frac{1}{2}-\sqrt{c}\right) \Lambda^{\sigma}+o\left(\Lambda^{\sigma}\right)
$$

holds as $\Lambda \rightarrow \infty$.

Proof of Lemma 2.1. Note that one can always assume $I=(0, l)$, where $l>0$ denotes the length of the interval. First we deduce the estimate for $\sigma=1$ from Lemma 2.2. Assume $\Lambda \geq l^{-2}$ and apply Lemma 2.2 with $A=l \sqrt{\Lambda} / \pi$ to get

$$
\operatorname{Tr}\left(-\frac{d^{2}}{d t^{2}}-\Lambda\right)_{-}=\Lambda \sum_{k}\left(1-\frac{\pi^{2} k^{2}}{l^{2} \Lambda}\right)_{+} \leq \Lambda \frac{2}{3 \pi} \int_{1}^{l \sqrt{\Lambda}}\left(1-\frac{1}{s^{2}}\right)^{3 / 2} d s
$$

Substituting $s=2 t \sqrt{\Lambda}$, we find that

$$
\operatorname{Tr}\left(-\frac{d^{2}}{d t^{2}}-\Lambda\right)_{-} \leq \frac{4}{3 \pi} \int_{1 /(2 \sqrt{\Lambda})}^{l / 2}\left(\Lambda-\frac{1}{4 t^{2}}\right)^{3 / 2} d t=2 L_{1,1}^{\mathrm{cl}} \int_{0}^{l / 2}\left(\Lambda-\frac{1}{4 t^{2}}\right)_{+}^{3 / 2} d t
$$

holds for all $\Lambda \geq l^{-2}$. Note that this inequality is trivially true for $0<\Lambda<l^{-2}$, since the left hand side is zero. Finally, we use the identities

$$
\int_{0}^{l / 2}\left(\Lambda-\frac{1}{4 t^{2}}\right)_{+}^{3 / 2} d t=\int_{l / 2}^{l}\left(\Lambda-\frac{1}{4(l-t)^{2}}\right)_{+}^{3 / 2} d t=\frac{1}{2} \int_{0}^{l}\left(\Lambda-\frac{1}{4 \delta(t)^{2}}\right)_{+}^{3 / 2} d t
$$

to finish the proof for $\sigma=1$.

To deduce the claim for $\sigma>1$ we can apply a method from [1]. Writing

$$
\sum_{k}\left(\Lambda-\lambda_{k}\right)_{+}^{\sigma}=\frac{1}{B(2, \sigma-1)} \int_{0}^{\infty} \tau^{\sigma-2} \sum_{k}\left(\Lambda-\tau-\lambda_{k}\right)_{+} d \tau,
$$

we estimate

$$
\begin{aligned}
\operatorname{Tr}\left(-\frac{d^{2}}{d t^{2}}-\Lambda\right)_{-}^{\sigma} & \leq \frac{1}{B(2, \sigma-1)} L_{1,1}^{\mathrm{cl}} \int_{I} \int_{0}^{\infty} \tau^{\sigma-2}\left(\Lambda-\frac{1}{4 \delta(t)^{2}}-\tau\right)_{+}^{3 / 2} d \tau d t \\
& =L_{1,1}^{\mathrm{cl}} \frac{B(5 / 2, \sigma-1)}{B(2, \sigma-1)} \int_{I}\left(\Lambda-\frac{1}{4 \delta(t)^{2}}\right)_{+}^{\sigma+1 / 2} d t
\end{aligned}
$$

and the result follows from the identity $L_{1,1}^{\mathrm{cl}} B(5 / 2, \sigma-1)=L_{\sigma, 1}^{\mathrm{cl}} B(2, \sigma-1)$.

The claim, that the constant $1 / 4$ cannot be improved, follows from Lemma 2.3: for $c=1 / 4$ the leading term of the asymptotics in Lemma 2.3 vanishes. For any constant $c>1 / 4$ the leading term is negative, thus the estimate from Lemma 2.1 must fail in this case, for large values of $\Lambda$. 
Figure 1 illustrates the results of Lemma 2.1 and Lemma 2.3 for $l=\pi^{2}$ and $\sigma=1$ with the sharp constant $c=1 / 4$ : the function

$$
f(\Lambda)=L_{1,1}^{\mathrm{cl}} \int_{0}^{\pi}\left(\Lambda-\frac{1}{4 \delta(t)^{2}}\right)_{+}^{3 / 2} d t-\sum_{k}\left(\Lambda-k^{2}\right)_{+}
$$

is plotted for $1<\Lambda<112$, so that the first ten minima are shown.

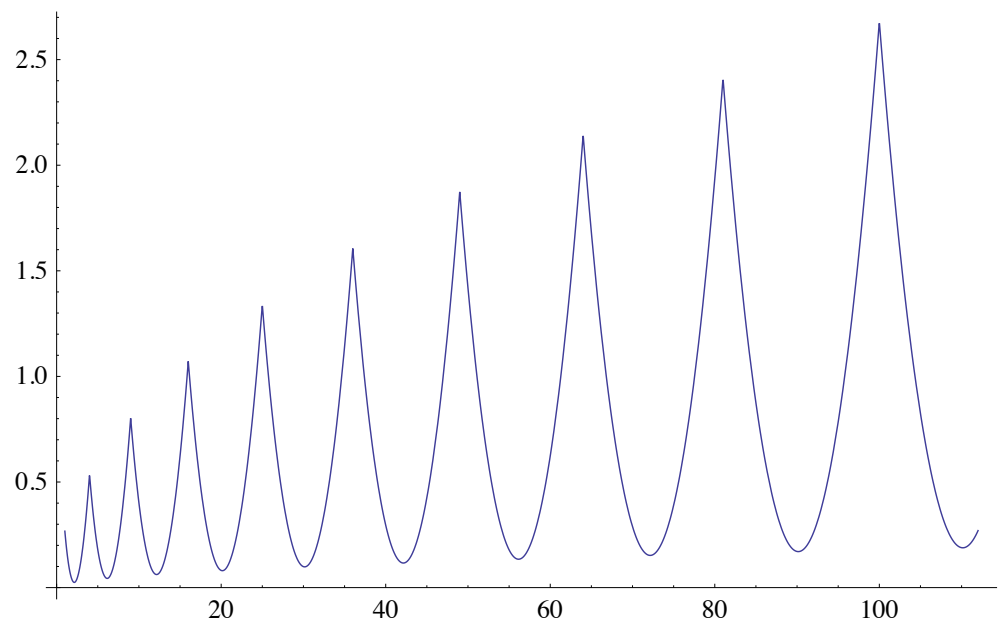

Figure 1. The function $f(\Lambda)$, illustrating the results from Section 2.

\section{Results in higher dimensions}

In this section we use the one-dimensional result to prove uniform eigenvalue estimates for the Dirichlet Laplace operator in bounded open sets in higher dimensions. These estimates - refinements of the Berezin inequality (1) - depend on the geometry of the set, in particular on properties of the boundary.

3.1. Arbitrary open sets. First we provide general estimates, valid for any open subset $\Omega \subset \mathbb{R}^{d}, d \geq 2$. Let

$$
\mathbb{S}^{d-1}=\left\{x \in \mathbb{R}^{d}:|x|=1\right\}
$$

denote the unit-sphere in $\mathbb{R}^{d}$. For an arbitrary direction $u \in \mathbb{S}^{d-1}$ and $x \in \Omega$ set

$$
\begin{aligned}
& \theta(x, u)=\inf \{t>0: x+t u \notin \Omega\}, \\
& d(x, u)=\inf \{\theta(x, u), \theta(x,-u)\},
\end{aligned}
$$


and

$$
l(x, u)=\theta(x, u)+\theta(x,-u) .
$$

Theorem 3.1. Let $\Omega \subset \mathbb{R}^{d}$ be an open set and let $u \in \mathbb{S}^{d-1}$ and $\sigma \geq 3 / 2$. Then for all $\Lambda>0$ the estimate

$$
\operatorname{Tr}(-\Delta-\Lambda)_{-}^{\sigma} \leq L_{\sigma, d}^{\mathrm{cl}} \int_{\Omega}\left(\Lambda-\frac{1}{4 d(x, u)^{2}}\right)_{+}^{\sigma+d / 2} d x
$$

holds true.

Remark 3.2. Let us define

$$
l_{0}=\inf _{u \in \Phi^{d-1}} \sup _{x \in \Omega} l(x, u) .
$$

Then Theorem 3.1 implies the following improvement of Melas-type: for $\sigma \geq 3 / 2$ and all $\Lambda>0$ the estimate

$$
\operatorname{Tr}(-\Delta-\Lambda)_{-}^{\sigma} \leq L_{\sigma, d}^{\mathrm{cl}}|\Omega|\left(\Lambda-\frac{1}{l_{0}^{2}}\right)_{+}^{\sigma+d / 2}
$$

holds. In convex domains $l_{0}$ is the minimal width of the domain, see [3]. In this case $1 / l_{0}^{2}$ is bounded from below by a multiple of $|\Omega|^{-2 / d},[35]$, while no such bound holds for the improving term $|\Omega| / I(\Omega)$ in Melas' inequality (4).

The proof of Theorem 3.1 relies on a lifting technique, which was introduced in [25], see also [26], [8], [33], and [9] for further developments and applications.

Proof of Theorem 3.1. We apply the argument used in [33] to reduce the problem to one-dimensional estimates. Fix a Cartesian coordinate system in $\mathbb{R}^{d}$, such that the given direction $u$ corresponds to the vector $(0, \ldots, 0,1)$.

For $x \in \mathbb{R}^{d}$ write $x=\left(x^{\prime}, t\right) \in \mathbb{R}^{d-1} \times \mathbb{R}$ and let $\nabla^{\prime}$ and $-\Delta^{\prime}$ denote the gradient and the Laplace operator in the first $d-1$ dimensions. Each section $\Omega\left(x^{\prime}\right)=\{t \in$ $\mathbb{R}:\left(x^{\prime}, t\right) \in \Omega$ \} consists of at most countably many open intervals $J_{k}\left(x^{\prime}\right) \subset \mathbb{R}$, $k=1, \ldots, N\left(x^{\prime}\right) \leq \infty$.

We consider the quadratic form of $-\Delta-\Lambda$ on functions $\varphi$ from the form core $C_{0}^{\infty}(\Omega)$ and write

$$
\|\nabla \varphi\|_{L^{2}(\Omega)}^{2}-\Lambda\|\varphi\|_{L^{2}(\Omega)}^{2}=\left\|\nabla^{\prime} \varphi\right\|_{L^{2}(\Omega)}^{2}+\int_{\mathbb{R}^{d}-1} d x^{\prime} \int_{\Omega\left(x^{\prime}\right)}\left(\left|\partial_{t} \varphi\right|^{2}-\Lambda|\varphi|^{2}\right) d t .
$$

The functions $\varphi\left(x^{\prime}, \cdot\right)$ satisfy Dirichlet boundary conditions at the endpoints of each interval $J_{k}\left(x^{\prime}\right)$ forming $\Omega\left(x^{\prime}\right)$, hence

$$
\int_{\Omega\left(x^{\prime}\right)}\left(\left|\partial_{t} \varphi\right|^{2}-\Lambda|\varphi|^{2}\right) d t=\sum_{k=1}^{N\left(x^{\prime}\right)} \int_{J_{k}\left(x^{\prime}\right)}\left(\left|\partial_{t} \varphi\right|^{2}-\Lambda|\varphi|^{2}\right) d t
$$




$$
\geq-\sum_{k=1}^{N\left(x^{\prime}\right)}\left\langle V_{k}\left(x^{\prime}\right) \varphi\left(x^{\prime}, \cdot\right), \varphi\left(x^{\prime}, \cdot\right)\right\rangle_{L^{2}\left(J_{k}\left(x^{\prime}\right)\right)}
$$

where the bounded, non-negative operators $V_{k}\left(x^{\prime}, \Lambda\right)=\left(-\partial_{t}^{2}-\Lambda\right)$ - are the negative parts of the Sturm-Liouville operators $-\partial_{t}^{2}-\Lambda$ with Dirichlet boundary conditions on $J_{k}\left(x^{\prime}\right)$. Let

$$
V\left(x^{\prime}, \Lambda\right)=\bigoplus_{k=1}^{N\left(x^{\prime}\right)} V_{k}\left(x^{\prime}, \Lambda\right)
$$

be the negative part of $-\partial_{t}^{2}-\Lambda$ on $\Omega\left(x^{\prime}\right)$ subject to Dirichlet boundary conditions on the endpoints of each interval $J_{k}\left(x^{\prime}\right), k=1, \ldots, N\left(x^{\prime}\right)$, that is on $\partial \Omega\left(x^{\prime}\right)$. Then

$$
\int_{\Omega\left(x^{\prime}\right)}\left(\left|\partial_{t} \varphi\right|^{2}-\Lambda|\varphi|^{2}\right) d t \geq-\left\langle V\left(x^{\prime}, \Lambda\right) \varphi\left(x^{\prime}, \cdot\right), \varphi\left(x^{\prime}, \cdot\right)\right\rangle_{L^{2}\left(\Omega\left(x^{\prime}\right)\right)}
$$

and consequently

$$
\|\nabla \varphi\|_{L^{2}(\Omega)}^{2}-\Lambda\|\varphi\|_{L^{2}(\Omega)}^{2} \geq\left\|\nabla^{\prime} \varphi\right\|_{L^{2}(\Omega)}^{2}-\int_{\mathbb{R}^{d-1}} d x^{\prime}\left\langle V \varphi\left(x^{\prime}, \cdot\right), \varphi\left(x^{\prime}, \cdot\right)\right\rangle_{L^{2}\left(\Omega\left(x^{\prime}\right)\right)} .
$$

Now we can extend this quadratic form by zero to $C_{0}^{\infty}\left(\mathbb{R}^{d} \backslash \partial \Omega\right)$, which is a form core for $\left(-\Delta_{\mathbb{R}^{d} \backslash \Omega}\right) \oplus\left(-\Delta_{\Omega}-\Lambda\right)$. This operator corresponds to the left hand side of the equality above, while the semi-bounded form on the right hand side is closed on the larger domain $H^{1}\left(\mathbb{R}^{d-1}, L^{2}(\mathbb{R})\right)$, where it corresponds to the operator

$$
-\Delta^{\prime} \otimes \mathbb{I}-V\left(x^{\prime}, \Lambda\right) \text { on } L^{2}\left(\mathbb{R}^{d-1}, L^{2}(\mathbb{R})\right) .
$$

Due to the positivity of $-\Delta_{\mathbb{R}} \backslash \Omega$ we can use the variational principle to deduce that for any $\sigma \geq 0$

$$
\begin{aligned}
\operatorname{Tr}\left(-\Delta_{\Omega}-\Lambda\right)_{-}^{\sigma} & =\operatorname{Tr}\left(\left(-\Delta_{\mathbb{R} d} \backslash \Omega\right.\right. \\
& \leq \operatorname{Tr}\left(-\Delta^{\prime} \otimes \mathbb{I}-V\left(x^{\prime}, \Lambda\right)\right)_{-}^{\sigma} .
\end{aligned}
$$

Now we apply sharp Lieb-Thirring inequalities [26] to the Schrödinger operator (10) with the operator-valued potential $-V\left(x^{\prime}, \Lambda\right)$ and obtain that for $\sigma \geq 3 / 2$

$$
\operatorname{Tr}\left(-\Delta_{\Omega}-\Lambda\right)_{-}^{\sigma} \leq L_{\sigma, d-1}^{\mathrm{cl}} \int_{\mathbb{R}^{d-1}} \operatorname{Tr} V\left(x^{\prime}, \Lambda\right)^{\sigma+(d-1) / 2} d x^{\prime} .
$$

To estimate the trace of the one-dimensional differential operator $V\left(x^{\prime}, \Lambda\right)$ we apply Lemma 2.1. Our choice of coordinate system implies that for $x=\left(x^{\prime}, t\right) \in$ $J_{k}\left(x^{\prime}\right)$ the distance of $t$ to the boundary of the interval $J_{k}\left(x^{\prime}\right)$ is given by $d(x, u)$. Hence, Lemma 2.1 implies

$$
\operatorname{Tr} V\left(x^{\prime}, \Lambda\right)^{\sigma+(d-1) / 2}=\sum_{k=1}^{N\left(x^{\prime}\right)} \operatorname{Tr}\left(-\left.\frac{d^{2}}{d t^{2}}\right|_{J_{k}\left(x^{\prime}\right)}-\Lambda\right)_{-}^{\sigma+(d-1) / 2}
$$




$$
\leq L_{\sigma+(d-1) / 2,1}^{\mathrm{cl}} \int_{\Omega\left(x^{\prime}\right)}\left(\Lambda-\frac{1}{4 d\left(\left(x^{\prime}, t\right), u\right)}\right)_{+}^{\sigma+d / 2} d t
$$

and the result follows from (11) and the identity $L_{\sigma, d-1}^{\mathrm{cl}} L_{\sigma+(d-1) / 2,1}^{\mathrm{cl}}=L_{\sigma, d}^{\mathrm{cl}}$.

We proceed to analysing the geometric properties of (7). Note that the left hand side of (7) is independent of the choice of direction $u \in \mathbb{S}^{d-1}$, while the right hand side depends on $u$ and therefore on the geometry of $\Omega$. For a given set $\Omega$ one can minimise the right hand side in $u \in \mathbb{S}^{d-1}$. The result, however, depends on the geometry of $\Omega$ in a rather tricky way. In order to make this geometric dependence more transparent, we average the right hand side of (7) over $u \in \mathbb{S}^{d-1}$. Even though the resulting bound is - in general - not as precise as (7), it allows a more appropriate geometric interpretation.

To analyse the effect of the boundary, one would like to estimate $d(x, u)$ in terms of the distance to the boundary. See [5], [6], and [18], where this approach is used to derive geometrical versions of Hardy's inequality. To avoid complications that arise, for example, if the complement of $\Omega$ contains isolated points, we use slightly different notions: for $x \in \Omega$ let

$$
\Omega(x)=\{y \in \Omega: x+t(y-x) \in \Omega, \forall t \in[0,1]\}
$$

be the part of $\Omega$ that "can be seen" from $x$ and let

$$
\delta(x)=\inf \{|y-x|: y \notin \overline{\Omega(x)}\}
$$

denote the distance to the exterior of $\Omega(x)$.

For fixed $\varepsilon>0$ put

$$
A_{\varepsilon}(x)=\left\{a \in \mathbb{R}^{d} \backslash \overline{\Omega(x)}:|x-a|<\delta(x)+\varepsilon\right\}
$$

and for $a \in A_{\varepsilon}(x)$ set $B_{x}(a)=\left\{y \in \mathbb{R}^{d}:|y-a|<|x-a|\right\}$ and

$$
\rho_{a}(x)=\frac{\left|B_{x}(a) \backslash \overline{\Omega(x)}\right|}{\omega_{d}|x-a|^{d}},
$$

where $\omega_{d}$ denotes the volume of the unit ball in $\mathbb{R}^{d}$. To get a result, independent of $a$ and $\varepsilon$, set

$$
\rho(x)=\inf _{\varepsilon>0} \sup _{a \in A_{\varepsilon}(x)} \rho_{a}(x) .
$$

Note that $\mathbb{R}^{d} \backslash \overline{\Omega(x)}$ is open, hence $\rho_{a}(x)>0$ and $\rho(x)>0$ hold for $x \in \Omega$. Finally, we define

$$
M_{\Omega}(\Lambda)=\int_{R_{\Omega}(\Lambda)} \rho(x) d x
$$

where $R_{\Omega}(\Lambda) \subset \Omega$ denotes the set $\{x \in \Omega: \delta(x)<1 /(4 \sqrt{\Lambda})\}$. The main result of this section allows a geometric interpretation of the remainder term. 
Theorem 3.3. Let $\Omega \subset \mathbb{R}^{d}$ be an open set with finite volume and $\sigma \geq 3 / 2$. Then for all $\Lambda>0$ we have

$$
\operatorname{Tr}(-\Delta-\Lambda)_{-}^{\sigma} \leq L_{\sigma, d}^{\mathrm{cl}}|\Omega| \Lambda^{\sigma+d / 2}-L_{\sigma, d}^{\mathrm{cl}} 2^{-d+1} \Lambda^{\sigma+d / 2} M_{\Omega}(\Lambda) .
$$

The function $\rho(x)$ depends on the behaviour of the boundary close to $x \in \Omega$. For example, $\rho(x)$ is small close to a cusp. On the other hand $\rho(x)$ is larger than $1 / 2$ in a convex domain. By definition, the function $M_{\Omega}(\Lambda)$ gives an average of this behaviour over $R_{\Omega}(\Lambda)$, which is like a tube of width $1 /(4 \sqrt{\Lambda})$ around the boundary.

Note that $M_{\Omega}(\Lambda)$ tends to zero as $\Lambda$ tends to infinity. This decay in $\Lambda$ is of the order $\left(\delta_{M}-d\right) / 2$, where $\delta_{M}$ denotes the interior Minkowski dimension of the boundary, see e.g. [24], [14], and [12] for definition and examples. If $d-1 \leq \delta_{M}<d$ and if the upper Minkowski content of the boundary is finite, then the second term of the asymptotic limit of the Riesz means equals $O\left(\Lambda^{\sigma+\delta_{M} / 2}\right)$ as $\Lambda \rightarrow \infty$, see [24]. Therefore the remainder term in (12) reflects the correct order of growth in the asymptotic limit.

In particular, if the dimension of the boundary equals $d-1$, we find

$$
M_{\Omega}(\Lambda)=|\partial \Omega| \Lambda^{-1 / 2}+o\left(\Lambda^{-1 / 2}\right)
$$

as $\Lambda \rightarrow \infty$ and the second term in (12) is in close correspondence with the asymptotic formula (3).

Proof of Theorem 3.3. We start from the result of Theorem 3.1 and average over all directions to get

$$
\operatorname{Tr}(-\Delta-\Lambda)_{-}^{\sigma} \leq L_{\sigma, d}^{\mathrm{cl}} \Lambda^{\sigma+d / 2} \int_{\Omega} \int_{\$ d-1}\left(1-\frac{1}{4 \Lambda d(x, u)^{2}}\right)_{+}^{\sigma+d / 2} d v(u) d x,
$$

where $d v(u)$ denotes the normed measure on $\mathbb{S}^{d-1}$.

For $x \in \Omega$ and $a \notin \overline{\Omega(x)}$ let $\Theta(x, a) \subset \mathbb{S}^{d-1}$ be the subset of all directions $u \in \mathbb{S}^{d-1}$, satisfying $x+s u \in B_{x}(a) \backslash \overline{\Omega(x)}$ for some $s>0$. For such $s$ we have

$$
s \leq 2|x-a| .
$$

By definition of $\rho_{a}(x)$ and $\Theta(x, a)$ we find

$$
\rho_{a}(x) \omega_{d}|x-a|^{d}=\left|B_{x}(a) \backslash \overline{\Omega(x)}\right| \leq \int_{\Theta(x, a)} d \nu(u) \omega_{d}(2|x-a|)^{d},
$$

hence

$$
\int_{\Theta(x, a)} d v(u) \geq 2^{-d} \rho_{a}(x) .
$$

Using (14) we also see that for $u \in \Theta(x, a)$ the estimate $d(x, u) \leq s \leq 2|x-a|$ holds. 
Now fix $\Lambda>0$ and choose $0<\varepsilon<1 /(4 \sqrt{\Lambda})$ and $a \in A_{\varepsilon}(x)$. By definition of $A_{\varepsilon}(x)$ it follows that for all $u \in \Theta(x, a)$

$$
d(x, u) \leq 2|x-a|<2(\delta(x)+\varepsilon) .
$$

The set $\Theta(x, a)$ must be contained in one hemisphere of $\rrbracket^{d-1}$ which we denote by $\mathbb{S}_{+}^{d-1}$. Using that $d(x, u)=d(x,-u)$ we estimate

$$
\begin{aligned}
\int_{\Phi^{d}-1}\left(1-\frac{1}{4 \Lambda d(x, u)^{2}}\right)_{+}^{\sigma+d / 2} d v(u) & =2 \int_{\Phi_{+}^{d-1}}\left(1-\frac{1}{4 \Lambda d(x, u)^{2}}\right)_{+}^{\sigma+d / 2} d v(u) \\
& \leq 1-2 \int_{\left\{u \in \Phi_{+}^{d-1}: d(x, u) \leq 1 /(2 \sqrt{\Lambda})\right\}} d v(u) .
\end{aligned}
$$

Assume that $\delta(x) \leq 1 /(4 \sqrt{\Lambda})-\varepsilon$. From (16) it follows that

$$
\Theta(x, a) \subset\left\{u \in \mathbb{S}_{+}^{d-1}: d(x, u) \leq 1 /(2 \sqrt{\Lambda})\right\},
$$

hence, using (15), we conclude

$$
\int_{\Phi d-1}\left(1-\frac{1}{4 \Lambda d(x, u)^{2}}\right)_{+}^{\sigma+d / 2} d v(u) \leq 1-2 \int_{\Theta(x, a)} d v(u) \leq 1-2^{1-d} \rho_{a}(x) .
$$

Since $a \in A_{\varepsilon}(x)$ was arbitrary we arrive at

$$
\int_{\Phi d-1}\left(1-\frac{1}{4 \Lambda d(x, u)^{2}}\right)_{+}^{\sigma+d / 2} d v(u) \leq 1-2^{1-d} \rho(x),
$$

for all $x \in \Omega$ with $\delta(x) \leq 1 /(4 \sqrt{\Lambda})-\varepsilon$ and we can take the limit $\varepsilon \rightarrow 0$.

It follows that

$$
\begin{aligned}
\int_{\Omega} \int_{\Phi d-1}(1- & \left.\frac{1}{4 \Lambda d(x, u)^{2}}\right)_{+}^{\sigma+d / 2} d \nu(u) d x \\
& \leq|\Omega|-2^{1-d} \int_{\{x \in \Omega: \delta(x)<1 /(4 \sqrt{\Lambda})\}} \rho(x) d x
\end{aligned}
$$

and inserting this into (13) yields the claimed result.

3.2. Convex domains. If $\Omega \subset \mathbb{R}^{d}$ is convex, we have $\Omega(x)=\Omega$ and

$$
\rho(x)>1 / 2
$$

for all $x \in \Omega$. Thus we can simplify the remainder term, by estimating $M_{\Omega}(\Lambda)$.

Corollary 3.4. Let $\Omega \subset \mathbb{R}^{d}$ be a bounded, convex domain and let $\Omega_{t}=\{x \in$ $\Omega: \delta(x)>t\}$ be the inner parallel set of $\Omega$. Then for $\sigma \geq 3 / 2$ and all $\Lambda>0$ we have

$$
\operatorname{Tr}(-\Delta-\Lambda)_{-}^{\sigma} \leq L_{\sigma, d}^{\mathrm{cl}}|\Omega| \Lambda^{\sigma+d / 2}-L_{\sigma, d}^{\mathrm{cl}} 2^{-d-2}\left|\partial \Omega_{1 /(4 \sqrt{\Lambda})}\right| \Lambda^{\sigma+(d-1) / 2} .
$$


Proof. Inserting (17) into the definition of $M_{\Omega}(\Lambda)$ yields

$$
\begin{aligned}
M_{\Omega}(\Lambda)>\frac{1}{2} \int_{R_{\Omega}(\Lambda)} d x & =\frac{1}{2} \int_{\{x \in \Omega: \delta(x)<1 /(4 \sqrt{\Lambda})\}} d x \\
& =\frac{1}{2} \int_{0}^{1 /(4 \sqrt{\Lambda})}\left|\partial \Omega_{t}\right| d t
\end{aligned}
$$

In view of (12) estimating

$$
\int_{0}^{1 /(4 \sqrt{\Lambda})}\left|\partial \Omega_{t}\right| d t \geq \frac{1}{4 \sqrt{\Lambda}}\left|\partial \Omega_{1 /(4 \sqrt{\Lambda})}\right|
$$

proves the claim.

If more is known about the geometry of $\Omega$ we can refine the estimate.

Corollary 3.5. Let $\Omega \subset \mathbb{R}^{d}$ be a bounded, convex domain with smooth boundary and assume that the curvature of $\partial \Omega$ is bounded from above by $1 / R$. Then for $\sigma \geq 3 / 2$ and all $\Lambda>0$ we have

$$
\begin{aligned}
\operatorname{Tr}(-\Delta-\Lambda)_{-}^{\sigma} \leq & L_{\sigma, d}^{\mathrm{cl}}|\Omega| \Lambda^{\sigma+d / 2} \\
& -L_{\sigma, d}^{\mathrm{cl}} 2^{-d-2}|\partial \Omega| \Lambda^{\sigma+(d-1) / 2} \int_{0}^{1}\left(1-\frac{d-1}{4 R \sqrt{\Lambda}} s\right)_{+} d s
\end{aligned}
$$

Note that one can estimate the integral in the remainder term to get the simplified bound

$$
\operatorname{Tr}(-\Delta-\Lambda)_{-}^{\sigma} \leq L_{\sigma, d}^{\mathrm{cl}}|\Omega| \Lambda^{\sigma+d / 2}-L_{\sigma, d}^{\mathrm{cl}} 2^{-d-2}|\partial \Omega| \Lambda^{\sigma+(d-1) / 2}\left(1-\frac{d-1}{8 R \sqrt{\Lambda}}\right)_{+} .
$$

Proof of Corollary 3.5. To estimate $M_{\Omega}(\Lambda)$ we can use Steiner's Theorem, see [15] and [32], namely

$$
\left|\partial \Omega_{t}\right| \geq\left(1-\frac{d-1}{R} t\right)_{+}|\partial \Omega| .
$$

From (18) it follows that

$$
M_{\Omega}(\Lambda)>\frac{1}{2}|\partial \Omega| \int_{0}^{1 /(4 \sqrt{\Lambda})}\left(1-\frac{d-1}{R} t\right)_{+} d t=\frac{|\partial \Omega|}{8 \sqrt{\Lambda}} \int_{0}^{1}\left(1-\frac{d-1}{4 R \sqrt{\Lambda}} s\right)_{+} d s
$$

Inserting this into (12) completes the proof. 


\section{Lower bounds on individual eigenvalues}

In order to further estimate the remainder terms, in particular to show that the remainder is negative for all $\Lambda \geq \lambda_{1}(\Omega)$ one needs suitable bounds on the ground state $\lambda_{1}(\Omega)$. We point out the following consequence of the proof of Theorem 3.1 which might be of independent interest.

Corollary 4.1. For any open set $\Omega \subset \mathbb{R}^{d}$ the estimate

$$
\lambda_{1}(\Omega) \geq \frac{\pi^{2}}{l_{0}^{2}}
$$

holds, where $l_{0}$ is given in (8).

Remark 4.2. One can compare this bound with

$$
\lambda_{1}(\Omega) \geq \inf _{x \in \Omega}\left(\frac{d}{4 m(x)^{2}}\right),
$$

see [5]. Here $m(x)^{-2}$ denotes the average of $d(x, u)^{-2}$ over all directions $u \in \mathbb{S}^{d-1}$. The relation between these bounds depends on the geometry of $\Omega$. In particular, the bound from Corollary 4.1 is stronger, if the width of $\Omega$ is small along an arbitrary direction $u \in \mathbb{S}^{d-1}$ such that $l_{0}$ is small enough.

Proof of Corollary 4.1. Fix $\varepsilon>0$ and choose a direction $u_{0} \in \mathbb{S}^{d-1}$, such that $\sup _{x \in \Omega} l\left(x, u_{0}\right)<l_{0}+\varepsilon$. We write $x=\left(x^{\prime}, t\right) \in \mathbb{R}^{d-1} \times \mathbb{R}$, where the $t$-axes is chosen in the direction of $u_{0}$. Let us recall inequality (11) from the proof of Theorem 3.1: for any $\sigma \geq 3 / 2$

$$
\operatorname{Tr}(-\Delta-\Lambda)_{-}^{\sigma} \leq L_{\sigma, d-1}^{\mathrm{cl}} \int_{\mathbb{R}^{d-1}} \operatorname{Tr} V\left(x^{\prime}, \Lambda\right)^{\sigma+(d-1) / 2} d x^{\prime},
$$

where $V\left(x^{\prime}, \Lambda\right)$ denotes the negative part of the operator $-\partial_{t}^{2}-\Lambda$ on $\Omega\left(x^{\prime}\right)=$ $\cup_{k=1}^{N\left(x^{\prime}\right)} J_{k}\left(x^{\prime}\right)$ with Dirichlet boundary conditions at the endpoints of each interval $J_{k}\left(x^{\prime}\right)$. This inequality can be rewritten as

$$
\operatorname{Tr}(-\Delta-\Lambda)_{-}^{\sigma} \leq L_{\sigma, d-1}^{\mathrm{cl}} \int_{\mathbb{R}^{d}-1} \sum_{k=1}^{N\left(x^{\prime}\right)} \sum_{j \in \mathbb{N}}\left(\Lambda-\frac{\pi^{2} j^{2}}{\left|J_{k}\left(x^{\prime}\right)\right|^{2}}\right)_{+}^{\sigma+(d-1) / 2} d x^{\prime} .
$$

Our choice of coordinate system implies $\left|J_{k}\left(x^{\prime}\right)\right| \leq \sup _{x \in \Omega} l\left(x, u_{0}\right)<l_{0}+\varepsilon$ for all $k=1, \ldots, N\left(x^{\prime}\right)$ and all $x^{\prime} \in \mathbb{R}^{d-1}$. It follows that the right hand side of the inequality above is zero for all $\Lambda \leq \pi^{2} /\left(l_{0}+\varepsilon\right)^{2}$. Thus by taking the limit $\varepsilon \rightarrow 0$ we find

$$
\sum_{n \in \mathbb{N}}\left(\Lambda-\lambda_{n}\right)_{+}^{\sigma}=\operatorname{Tr}(-\Delta-\Lambda)_{-}^{\sigma}=0
$$

for all $\Lambda \leq \pi^{2} / l_{0}^{2}$ and $\lambda_{1} \geq \pi^{2} / l_{0}^{2}$ follows. 
From (9) we obtain similar bounds on higher eigenvalues using a method introduced in [25].

Corollary 4.3. For any open set $\Omega \subset \mathbb{R}^{d}$ with finite volume and any $k \in \mathbb{N}$ the estimate

$$
\lambda_{k}(\Omega) \geq C_{d}\left(\frac{12}{\pi}\right)^{1 / d} \frac{d}{(d+3)^{1+1 / d}}\left(\frac{\Gamma((d+3) / 2)}{\Gamma(d / 2+1)}\right)^{2 / d} \frac{k^{2 / d}}{|\Omega|^{2 / d}}+\frac{1}{l_{0}^{2}}
$$

holds, with

$$
C_{d}=4 \pi \Gamma(d / 2+1)^{2 / d}
$$

Proof. Let $N(\Lambda)=\operatorname{Tr}(-\Delta-\Lambda)_{-}^{0}$ denote the counting function of the eigenvalues below $\Lambda>0$. In [25] it is shown that for $\sigma>0$, and all $\Lambda>0, \tau>0$

$$
N(\Lambda) \leq(\tau \Lambda)^{-\sigma} \operatorname{Tr}(-\Delta-(1+\tau) \Lambda)_{-}^{\sigma} .
$$

If we apply this inequality with $\sigma=3 / 2$, we can use (9) to estimate

$$
N(\Lambda) \leq L_{3 / 2, d}^{\mathrm{cl}}|\Omega| \Lambda^{d / 2} \frac{(1+\tau)^{(d+3) / 2}}{\tau^{3 / 2}}\left(1-\frac{1}{\Lambda(1+\tau) l_{0}^{2}}\right)_{+}^{(d+3) / 2} .
$$

Minimising the right hand side in $\tau>0$ yields $\tau_{\min }=3\left(\Lambda l_{0}^{2}-1\right) /\left(d \Lambda l_{0}^{2}\right)$ and inserting this we find

$$
N(\Lambda) \leq L_{3 / 2, d}^{\mathrm{cl}}|\Omega| \frac{(d+3)^{(d+3) / 2}}{3^{3 / 2} d^{d / 2}}\left(\Lambda-\frac{1}{l_{0}^{2}}\right)_{+}^{d / 2} .
$$

This is equivalent to the claimed result.

Remark 4.4. Applying the same method to (1) with $\sigma=1$ we recover the LiYau inequality (5). In the proof of Corollary 4.3 we have to start from $\sigma=3 / 2$, therefore the result is not strong enough to improve (5) in general, i.e. the coefficient of $k^{2 / d} /|\Omega|^{2 / d}$ in Corollary 4.3 is less than $C_{d} \cdot d /(d+2)$ for all $d \geq 2$. However, one gets an improvement of (5) for low eigenvalues whenever $l_{0}$ is small.

\section{Further improvements in dimension 2}

In this section we further improve Corollary 3.5 and generalise it to a large class of bounded convex domains $\Omega \subset \mathbb{R}^{2}$. Here we do not require smoothness, therefore we cannot use (19) to estimate inner parallels of the boundary. To find a suitable substitute let $w$ denote the minimal width of $\Omega$ and note that for $l_{0}$ given in (8) the identity

$$
w=l_{0}
$$


holds true, see e.g. [3]. In the remainder of this section we assume that for all $t>0$

$$
\left|\partial \Omega_{t}\right| \geq\left(1-\frac{3 t}{w}\right)_{+}|\partial \Omega|
$$

This is true for a large class of convex domains, including the circle, regular polygons and arbitrary triangles and equality holds for the equilateral triangle. Actually we conjecture that (21) holds true for all bounded convex domains in $\mathbb{R}^{2}$.

Furthermore we need a lower bound on the ground state. From Corollary 4.1 we obtain that for all convex domains $\Omega \subset \mathbb{R}^{2}$

$$
\lambda_{1}(\Omega) \geq \frac{\pi^{2}}{w^{2}}
$$

holds. One should mention, that this follows immediately from the variational principle and the fact that $\lambda_{1}(\Omega)=\pi^{2} / w^{2}$ if $\Omega$ is the infinite strip with width $w$. Moreover, the same estimate can be obtained from the inequality $\lambda_{1}(\Omega) \geq \pi^{2} /\left(4 r_{i n}^{2}\right)$, see [30], where $r_{i n}$ is the inradius of $\Omega$.

Using (21) and (22) we can derive a more precise version of Theorem 3.3, where the correction term depends only on $|\partial \Omega|$ and is apart from that independent of the geometry of $\Omega$.

Theorem 5.1. Let $\Omega \subset \mathbb{R}^{2}$ be a bounded, convex domain, satisfying (21). Then for $\sigma \geq 3 / 2$ we have

$$
\operatorname{Tr}(-\Delta-\Lambda)_{-}^{\sigma}=0 \quad \text { if } \Lambda \leq \pi^{2} / w^{2}
$$

and

$$
\operatorname{Tr}(-\Delta-\Lambda)_{-}^{\sigma} \leq L_{\sigma, 2}^{\mathrm{cl}}|\Omega| \Lambda^{\sigma+1}-C_{\mathrm{co}} L_{\sigma, 1}^{\mathrm{cl}}|\partial \Omega| \Lambda^{\sigma+1 / 2} \quad \text { if } \Lambda>\pi^{2} / w^{2},
$$

with a constant

$$
C_{\mathrm{co}} \geq \frac{11}{9 \pi^{2}}-\frac{3}{20 \pi^{4}}-\frac{2}{5 \pi^{2}} \ln \left(\frac{4 \pi}{3}\right)>0.0642 .
$$

Proof. The first claim follows directly from (22), thus we can assume $\Lambda>\pi^{2} / w^{2}$. First we prove the result for $\sigma=3 / 2$. Again we can start from (13) and we need to estimate $d(x, u)$ in terms of $\delta(x)$, which is just the distance to the boundary, since $\Omega$ is convex.

Fix $x \in \Omega$. Since $\Omega$ is convex and smooth we can choose $u_{0} \in \mathbb{S}^{d-1}$, such that $d\left(x, u_{0}\right)=\delta(x)$. We can assume $u_{0}=(1,0, \ldots, 0)$ and put $\mathbb{S}_{+}^{d-1}=\{u \in$ $\left.\mathbb{R}^{d}: u_{1}>0\right\}$.

Let $a$ be the intersection point of the semi-axes $\left\{x+t u_{0}, t>0\right\}$ with $\partial \Omega$ and for arbitrary $u \in \mathbb{S}_{+}^{d-1}$ let $b_{u}$ be the intersection point of $\{x+t u, t>0\}$ with the plane through $a$, orthogonal to $u_{0}$. We find $d(x, u) \leq\left|x-b_{u}\right|$ and if $\theta_{u}$ denotes the angle between $u_{0}$ and $u$, we find

$$
d(x, u) \leq\left|x-b_{u}\right|=\frac{|x-a|}{\cos \theta_{u}}=\frac{\delta(x)}{\cos \theta_{u}} .
$$


Using (13) and taking into account that $d(x, u)=d(x,-u)$ we get

$$
\operatorname{Tr}(-\Delta-\Lambda)_{-}^{3 / 2} \leq L_{3 / 2,2}^{\mathrm{cl}} \Lambda^{5 / 2} \int_{\Omega} \frac{2}{\pi} \int_{\theta_{0}}^{\pi / 2}\left(1-\frac{\cos ^{2}(\theta)}{4 \Lambda \delta(x)^{2}}\right)^{5 / 2} d \theta d x,
$$

where $\theta_{0}=0$ if $\delta(x) \geq 1 /(2 \sqrt{\Lambda})$ and $\theta_{0}=\arccos (2 \delta(x) \sqrt{\Lambda})$ if $\delta(x)<1 /(2 \sqrt{\Lambda})$. To obtain a simple bound one could estimate

$$
\left(1-\frac{\cos ^{2}(\theta)}{4 \Lambda \delta(x)^{2}}\right)^{5 / 2} \leq 1-\frac{\cos ^{2}(\theta)}{4 \Lambda \delta(x)^{2}} .
$$

However, to get a result as precise as possible we use

$$
\left(1-\frac{\cos ^{2}(\theta)}{4 \Lambda \delta(x)^{2}}\right)^{5 / 2} \leq 1-\frac{\cos ^{2}(\theta)}{2 \Lambda \delta(x)^{2}}+\frac{\cos ^{4}(\theta)}{16 \Lambda^{2} \delta(x)^{4}}
$$

and get

$$
\operatorname{Tr}(-\Delta-\Lambda)_{-}^{3 / 2} \leq \frac{\Lambda^{5 / 2}}{5 \pi^{2}} \int_{\Omega} \int_{\theta_{0}}^{\pi / 2}\left(1-\frac{\cos ^{2}(\theta)}{2 \Lambda \delta(x)^{2}}+\frac{\cos ^{4}(\theta)}{16 \Lambda^{2} \delta(x)^{4}}\right) d \theta d x .
$$

Set $u_{0}=\min (1,2 \delta(x) \sqrt{\Lambda})$ and calculate

$$
\begin{aligned}
\int_{\theta_{0}}^{\pi / 2}\left(1-\frac{\cos ^{2}(\theta)}{2 \Lambda \delta(x)^{2}}+\frac{\cos ^{4}(\theta)}{16 \Lambda^{2} \delta(x)^{4}}\right) d \theta= & \frac{\pi}{2}-\theta_{0}-\frac{\arcsin \left(u_{0}\right)-u_{0} \sqrt{1-u_{0}^{2}}}{4 \Lambda \delta(x)^{2}} \\
& +\frac{3 \arcsin \left(u_{0}\right)-u_{0}\left(2 u_{0}^{2}+3\right) \sqrt{1-u_{0}^{2}}}{128 \Lambda^{2} \delta(x)^{4}} .
\end{aligned}
$$

Inserting this back into (23) yields

$$
\operatorname{Tr}(-\Delta-\Lambda)_{-}^{3 / 2} \leq \frac{1}{10 \pi}|\Omega| \Lambda^{5 / 2}-\Lambda^{5 / 2}\left(I_{1}+I_{2}\right),
$$

where

$$
\left.I_{1}=\int_{\{\delta(x) \geq 1 /(2 \sqrt{\Lambda})}\right\} \frac{1}{10 \pi}\left(\frac{1}{4 \Lambda \delta(x)^{2}}-\frac{3}{128 \Lambda^{2} \delta(x)^{4}}\right) d x
$$

and

$$
\begin{aligned}
I_{2}=\int_{\{\delta(x)<1 /(2 \sqrt{\Lambda})\}} \frac{1}{5 \pi^{2}}( & \arccos (2 \sqrt{\Lambda} \delta(x))+\frac{\arcsin (2 \sqrt{\Lambda} \delta(x))}{4 \Lambda \delta(x)^{2}} \\
& -\frac{\sqrt{1-4 \Lambda \delta(x)^{2}}}{2 \sqrt{\Lambda} \delta(x)}-\frac{3 \arcsin (2 \sqrt{\Lambda} \delta(x))}{128 \Lambda^{2} \delta(x)^{4}} \\
& \left.+\frac{\left(8 \Lambda \delta(x)^{2}+3\right) \sqrt{1-4 \Lambda \delta(x)^{2}}}{64 \Lambda^{3 / 2} \delta(x)^{3}}\right) d x
\end{aligned}
$$


First we turn to

$$
I_{1}=\frac{1}{10 \pi} \int_{1 /(2 \sqrt{\Lambda})}^{\infty}\left|\partial \Omega_{t}\right|\left(\frac{1}{4 \Lambda t^{2}}-\frac{3}{128 \Lambda^{2} t^{4}}\right) d t .
$$

Note that the term in brackets is positive, thus after substituting $s=2 \sqrt{\Lambda} t$ we can use (21) and $\Lambda>\pi^{2} / w^{2}$ to obtain

$$
\begin{aligned}
I_{1} & \geq \frac{1}{20 \pi} \frac{|\partial \Omega|}{\sqrt{\Lambda}} \int_{1}^{\infty}\left(1-\frac{3 s}{2 \pi}\right)_{+}\left(\frac{1}{s^{2}}-\frac{3}{8 s^{4}}\right) d s \\
& =\frac{1}{20 \pi} \frac{|\partial \Omega|}{\sqrt{\Lambda}}\left(\frac{7}{8}-\frac{39}{32 \pi}-\frac{27}{128 \pi^{3}}-\frac{3}{2 \pi} \ln \left(\frac{2 \pi}{3}\right)\right) .
\end{aligned}
$$

Similarly we can treat $I_{2}$ and get

$$
I_{2} \geq \frac{1}{10 \pi^{2}} \frac{|\partial \Omega|}{\sqrt{\Lambda}}\left(\frac{557}{192}-\frac{7 \pi}{16}-\frac{3}{2 \pi} \int_{0}^{1} \frac{\arcsin (s)}{s} d s\right)
$$

In view of $L_{3 / 2,1}^{\mathrm{cl}}=3 / 16$ we can write

$$
I_{1}+I_{2} \geq L_{3 / 2,1}^{\mathrm{cl}} \frac{|\partial \Omega|}{\sqrt{\Lambda}}\left(\frac{11}{9 \pi^{2}}-\frac{3}{20 \pi^{4}}-\frac{2}{5 \pi^{2}} \ln \left(\frac{2 \pi}{3}\right)-\frac{2}{5 \pi^{2}} \ln (2)\right)
$$

and inserting this into (24) yields the claim in the case $\sigma=3 / 2$.

To prove the estimate for $\sigma>3 / 2$ we again refer to [1] and use the identity

$$
\operatorname{Tr}(-\Delta-\Lambda)_{-}^{\sigma}=\frac{1}{B(\sigma-3 / 2,5 / 2)} \int_{0}^{\infty} \tau^{\sigma-5 / 2} \operatorname{Tr}(-\Delta-(\Lambda-\tau))_{-}^{3 / 2} d \tau,
$$

from which the general result follows.

Now we can apply the same arguments that lead to Corollary 4.3 to derive lower bounds on individual eigenvalues.

Corollary 5.2. Let $\Omega \subset \mathbb{R}^{2}$ be a bounded, convex domain, satisfying (21). Then for $k \in \mathbb{N}$ and any $\alpha \in(0,1)$ the estimate

$$
\begin{aligned}
\frac{\lambda_{k}(\Omega)}{1-\alpha} \geq & 10 \pi \alpha^{3 / 2} \frac{k}{|\Omega|}+\frac{15 \pi C_{\mathrm{co}}}{8} \frac{|\partial \Omega|}{|\Omega|} \sqrt{10 \pi \alpha^{3 / 2} \frac{k}{|\Omega|}+\frac{225 \pi^{2} C_{\mathrm{co}}^{2}}{256} \frac{|\partial \Omega|^{2}}{|\Omega|^{2}}} \\
& +\frac{225 \pi^{2} C_{\mathrm{co}}^{2}}{128} \frac{|\partial \Omega|^{2}}{|\Omega|^{2}}
\end{aligned}
$$

holds, with the constant $C_{\mathrm{co}}$ given in Theorem 5.1 . 
Proof. Applying (20) and Theorem 5.1 with $\sigma=3 / 2$ yields

$$
N(\Lambda) \leq L_{3 / 2,2}^{\mathrm{cl}}|\Omega| \Lambda \frac{(1+\tau)^{5 / 2}}{\tau^{3 / 2}}-C_{\mathrm{co}} L_{3 / 2,1}^{\mathrm{cl}}|\partial \Omega| \sqrt{\Lambda} \frac{(1+\tau)^{2}}{\tau^{3 / 2}}
$$

for any $\tau>0$ and $\Lambda>\pi^{2} / w^{2}$. With $\tau=\alpha /(1-\alpha), \alpha \in(0,1)$, this is equivalent to the claimed estimate.

Remark 5.3. Given a fixed ratio $|\partial \Omega| /|\Omega|$ one can optimise the foregoing estimate in $\alpha \in(0,1)$, depending on $k \in \mathbb{N}$. As mentioned in the remark after Corollary 4.3, the result cannot improve the $\mathrm{Li}-$ Yau inequality (5) in general, since we have to apply (20) with $\sigma=3 / 2$ instead of $\sigma=1$. However, the estimates obtained from Corollary 5.2 are stronger than (5) for low eigenvalues and the improvements depend on the ratio $|\partial \Omega| /|\Omega|$.

In particular, one can use the isoperimetric inequality, namely $|\partial \Omega| \geq 2(\pi|\Omega|)^{1 / 2}$ for all $\Omega \subset \mathbb{R}^{2}$, to derive general improvements of the Li-Yau inequality (5) for low eigenvalues. From (5) we get

$$
\lambda_{k}(\Omega) \geq \frac{2 \pi k}{|\Omega|}
$$

and one can supplement these estimates with the Krahn-Szegö inequality [17]

$$
\lambda_{2}(\Omega) \geq \frac{2 \pi j_{0,1}^{2}}{|\Omega|}
$$

Optimising the estimate of Corollary 5.2 with $|\partial \Omega|=2(\pi|\Omega|)^{1 / 2}$, we find that for any convex domain, satisfying (21),

$$
\begin{aligned}
& \lambda_{6}(\Omega)>\frac{40.50}{|\Omega|}>\frac{12 \pi}{|\Omega|}>\frac{2 \pi j_{0,1}^{2}}{|\Omega|}, \\
& \lambda_{7}(\Omega)>\frac{46.74}{|\Omega|}>\frac{14 \pi}{|\Omega|}, \\
& \ldots, \\
& \lambda_{23}(\Omega)>\frac{144.58}{|\Omega|}>\frac{46 \pi}{|\Omega|} .
\end{aligned}
$$

In this way we can improve (5) and (25) in convex domains for all eigenvalues $\lambda_{k}(\Omega)$ with $6 \leq k \leq 23$.

Finally let us make a remark about the square $Q_{l}=(0, l) \times(0, l) \subset \mathbb{R}^{2}, l>0$. Using the methods introduced in Section 2 one can establish the following twodimensional version of Lemma 2.1: choose a coordinate system $\left(x_{1}, x_{2}\right) \in \mathbb{R}^{2}$ with axes parallel to the sides of the square and for $x \in Q_{l}$ put

$$
\delta\left(x_{i}\right)=\min \left(x_{i}, l-x_{i}\right), \quad i=1,2 .
$$


Then for $\sigma \geq 1$ and all $\Lambda>0$ the estimate

$$
\begin{aligned}
\operatorname{Tr}(-\Delta-\Lambda)_{-}^{\sigma} & =\sum_{m, n \in \mathbb{N}}\left(\Lambda-\frac{\pi^{2}}{l^{2}}\left(n^{2}+m^{2}\right)\right)_{+}^{\sigma} \\
& \leq L_{\sigma, 2}^{\mathrm{cl}} \int_{0}^{l} \int_{0}^{l}\left(\Lambda-C_{s q}\left(\frac{1}{\delta\left(x_{1}\right)}+\frac{1}{\delta\left(x_{2}\right)}\right)^{2}\right)_{+}^{\sigma+1} d x_{1} d x_{2}
\end{aligned}
$$

holds with a constant $C_{s q}>1 / 10$.

\section{A. Proof of Lemma 2.2 and Lemma 2.3}

A.1. Proof of Lemma 2.2. For $A \in \mathbb{R}$ let $\bar{A}$ and $\widetilde{A}$ denote the integer and fractional part of $A$ respectively. Then we can calculate

$$
\begin{aligned}
\sum_{k}\left(1-\frac{k^{2}}{A^{2}}\right)_{+} & =\sum_{k=1}^{\bar{A}}\left(1-\frac{k^{2}}{A^{2}}\right)=\bar{A}-\frac{1}{A^{2}}\left(\frac{\bar{A}^{3}}{3}+\frac{\bar{A}^{2}}{2}+\frac{\bar{A}}{6}\right) \\
& =\frac{2 A}{3}-\frac{1}{2}-\frac{1}{6 A}+\widetilde{A}(1-\widetilde{A}) \frac{1}{A}+\widetilde{A}\left(1-3 \widetilde{A}+2 \widetilde{A}^{2}\right) \frac{1}{6 A^{2}}
\end{aligned}
$$

From $0 \leq \tilde{A}<1$ we conclude $\widetilde{A}(1-\widetilde{A}) \leq 1 / 4$ and $\widetilde{A}\left(1-3 \widetilde{A}+2 \widetilde{A}^{2}\right) \leq \sqrt{3} / 18$ and we get

$$
\sum_{k}\left(1-\frac{k^{2}}{A^{2}}\right)_{+} \leq \frac{2 A}{3}-\frac{1}{2}+\frac{1}{12 A}+\frac{\sqrt{3}}{108 A^{2}} .
$$

To estimate the right hand side of (6) note that

$$
\int\left(1-\frac{1}{s^{2}}\right)^{3 / 2} d s=\left(1+\frac{1}{2 s^{2}}\right) \sqrt{s^{2}-1}+\frac{3}{2} \arctan \left(\frac{1}{\sqrt{s^{2}-1}}\right),
$$

thus

$$
\frac{2}{3 \pi} \int_{1}^{\pi A}\left(1-\frac{1}{s^{2}}\right)^{3 / 2} d s=\frac{2 \pi^{2} A^{2}+1}{3 \pi^{2} A} \frac{\sqrt{\pi^{2} A^{2}-1}}{\pi A}+\frac{1}{\pi} \arctan \left(\frac{1}{\sqrt{\pi^{2} A^{2}-1}}\right)-\frac{1}{2} .
$$

Now we can insert the elementary estimates

$$
\arctan \left(\frac{1}{\sqrt{\pi^{2} A^{2}-1}}\right) \geq \frac{1}{\pi A}
$$

and

$$
\frac{\sqrt{\pi^{2} A^{2}-1}}{\pi A} \geq 1-\frac{1}{2 \pi^{2} A^{2}}-\frac{a}{A^{4}}
$$


both valid for $A \geq 2$, where we write $a=16-2 / \pi^{2}-8 \sqrt{4 \pi^{2}-1} / \pi$ for simplicity. We get

$$
\frac{2}{3 \pi} \int_{1}^{\pi A}\left(1-\frac{1}{s^{2}}\right)^{3 / 2} d s \geq \frac{2 A}{3}-\frac{1}{2}+\frac{1}{\pi^{2} A}-\frac{1}{6 \pi^{4} A^{3}}-\frac{a}{3}\left(\frac{2}{A^{3}}+\frac{1}{\pi^{2} A^{5}}\right)
$$

for all $A \geq 2$. From (26) and (27) we deduce that (6) holds true for all $A \geq 2$, since

$$
\left(\frac{1}{\pi^{2}}-\frac{1}{12}\right) A^{4}-\frac{\sqrt{3}}{108} A^{3}-\left(\frac{1}{6 \pi^{4}}+\frac{2 a}{3}\right) A^{2}-\frac{a}{3 \pi^{2}} \geq 0
$$

for all $A \geq 2$.

Note that (6) is trivial for $1 / \pi \leq A \leq 1$, since the left hand side equals zero. The remaining case $1 \leq A \leq 2$ can be checked by hand.

A.2. Proof of Lemma 2.3. We assume $I=(0, l)$, substitute $t=s \sqrt{c / \Lambda}$ and write

$$
\int_{0}^{l}\left(\Lambda-\frac{c}{\delta(t)^{2}}\right)_{+}^{\sigma+1 / 2} d t=2 \sqrt{c} \Lambda^{\sigma} \int_{1}^{l \sqrt{\Lambda} /(2 \sqrt{c})}\left(1-\frac{1}{s^{2}}\right)^{\sigma+1 / 2} d s
$$

The claim of the lemma follows, if we show that

$$
2 \sqrt{c} L_{\sigma, 1}^{\mathrm{cl}} \int_{1}^{l \sqrt{\Lambda} /(2 \sqrt{c})}\left(1-\frac{1}{s^{2}}\right)^{\sigma+1 / 2} d s-\sum_{k}\left(1-\frac{\pi^{2} k^{2}}{\Lambda l^{2}}\right)_{+}^{\sigma}=\frac{1}{2}-\sqrt{c}+o(1)
$$

as $\Lambda \rightarrow \infty$. With $A=l \sqrt{\Lambda} / \pi$ this is equivalent to

$$
2 \sqrt{c} L_{\sigma, 1}^{\mathrm{cl}} \int_{1}^{\pi A /(2 \sqrt{c})}\left(1-\frac{1}{s^{2}}\right)^{\sigma+1 / 2} d s-\sum_{k}\left(1-\frac{k^{2}}{A^{2}}\right)_{+}^{\sigma}=\frac{1}{2}-\sqrt{c}+o(1)
$$

as $A \rightarrow \infty$.

It is easy to see that

$$
\sum_{k}\left(1-\frac{k^{2}}{A^{2}}\right)_{+}^{\sigma}=\frac{A}{2} B\left(\sigma+1, \frac{1}{2}\right)-\frac{1}{2}+o(1)
$$

as $A \rightarrow \infty$. Moreover, we claim

$$
\int_{1}^{\pi A /(2 \sqrt{c})}\left(1-\frac{1}{s^{2}}\right)^{\sigma+1 / 2} d s=\frac{\pi A}{2 \sqrt{c}}+\frac{1}{2} B\left(-\frac{1}{2}, \sigma+\frac{3}{2}\right)+o(1)
$$

as $A \rightarrow \infty$ and (28) follows from $L_{\sigma, 1}^{\mathrm{cl}}=B(\sigma+1,1 / 2) /(2 \pi)$, if we can establish (29). Let us write

$$
\left(1-\frac{1}{s^{2}}\right)^{m}=\sum_{k \geq 0}(-1)^{k}\left(\begin{array}{c}
m \\
k
\end{array}\right) s^{-2 k}=\sum_{k \geq 0}\left(\begin{array}{c}
k-m-1 \\
k
\end{array}\right) s^{-2 k}
$$


for $m \geq 1$ and note that the sum is finite if $m \in \mathbb{N}$, while the sum converges uniformly on $s \in[1, \infty)$ if $m \notin \mathbb{N}$. Hence we have

$$
\int_{1}^{y}\left(1-\frac{1}{s^{2}}\right)^{m} d s=y+\sum_{k \geq 0}\left(\begin{array}{c}
k-m-1 \\
k
\end{array}\right) \frac{1}{2 k-1}+o(1)
$$

as $y \rightarrow \infty$. Using that

$$
\sum_{k \geq 0}\left(\begin{array}{c}
k-m-1 \\
k
\end{array}\right) \frac{1}{2 k-1}=\frac{1}{2} B\left(-\frac{1}{2}, m+1\right)
$$

we obtain

$$
\int_{1}^{y}\left(1-\frac{1}{s^{2}}\right)^{m} d s=y+\frac{1}{2} B\left(-\frac{1}{2}, m+1\right)+o(1)
$$

as $y \rightarrow \infty$, which is equivalent to (29). This finishes the proof of Lemma 2.3.

\section{References}

[1] M. Aizenman and E. H. Lieb, On semi-classical bounds for eigenvalues of Schrödinger operators. Phys. Lett. 66 (1978), 427-429. MR 0598768

[2] F. A. Berezin, Covariant and contravariant symbols of operators. Izv. Akad. Nauk SSSR Ser. Mat. 13 (1972), 1134-1167. MR 0350504

[3] T. Bonnesen and W.Fenchel, Theorie der konvexen Körper. Chelsea Publishing Company, New York, 1948.

[4] R. Courant and D. Hilbert, Methoden der mathematischen Physik. Springer, Berlin, 1924. JFM 50.0335.07

[5] E. B. Davies, Spectral theory and differential operators. Cambridge Stud. Adv. Math. 42, Cambridge University Press, Cambridge, 1995. MR 1349825

[6] E. B. Davies, A review of Hardy inequalities, The Maz'ya anniversary collection. Vol. 2, Oper. Theory Adv. Appl., vol. 110, Birkhäuser, Basel, 1999, pp. 55-67. MR 1747888 Zbl 0936.35121

[7] T. Ekholm and R. L. Frank, On Lieb-Thirring inequalities for Schrödinger operators with virtual level. Comm. Math. Phys. 264 (2006), 725-740. MR 2217288 Zbl 1106.81039

[8] P. Exner, H. Linde, and T. Weidl, Lieb-Thirring inequalities for geometrically induced bound states. Lett. Math. Phys. 70 (2004), no. 1, 83-95. MR 2107707 Zbl 1059.81039

[9] R. L. Frank and A. Laptev, Spectral inequalities for Schrödinger operators with surface potentials. Spectral theory of differential operators, Amer. Math. Soc. Transl. Ser. 2, vol. 225, Amer. Math. Soc., Providence, RI, 2008, pp. 91-102. MR 2509777 Zbl 1170.35486

[10] R. L. Frank, E. H. Lieb, and R. Seiringer, Hardy-Lieb-Thirring inequalities for fractional Schrödinger operators. J. Amer. Math. Soc. 21 (2008), no. 4, 925-950. MR 2425175 Zbl 1202.35146 
[11] J. K. Freericks, E. H. Lieb, and D. Ueltschi, Segregation in the Falicov-Kimball model, Comm. Math. Phys. 227 (2002), no. 2, 243-279. MR 1903646 Zbl 1004.82009

[12] J. Fleckinger, M. Levitin, and D. Vassiliev, Heat equation on the triadic von Koch snowflake: asymptotic and numerical analysis. Proc. London Math. Soc. 71 (1995), no. 3 , 372-396. MR 1337471 Zbl 0836.35058

[13] R. L.Frank, A simple proof of Hardy-Lieb-Thirring inequalities.Comm. Math.Phys. 290 (2009), no. 2, 789-800. MR 2525639 Zbl 1184.35121

[14] J. Fleckinger and D. G. Vassiliev, An example of a two-term asymptotics for the "counting function" of a fractal drum. Trans. Amer. Math. Soc. 337 (1993), no. 1, 99-116. MR 1176086 Zbl 0851.35102

[15] H. W. Guggenheimer, Differential geometry. Dover, New York, 1977. MR 0493768 Zbl 0357.53002

[16] L. Geisinger and T. Weidl, Universal bounds for traces of the Dirichlet Laplace operator. J. Lond. Math. Soc. 82 (2010), no. 2, 395-419. MR 2725046 Zbl 05797872

[17] A. Henrot, Extremum problems for eigenvalues of elliptic operators, Frontiers in Mathematics, Birkäuser Verlag. Basel, 2006. MR 2251558 Zbl 1109.35081

[18] M.Hoffmann-Ostenhof, T. Hoffmann-Ostenhof, and A. Laptev, A geometrical version of Hardy's inequality. J. Funct. Anal. 189 (2002), 539-548. MR 1892180 Zbl 1012.26011

[19] L. Hörmander, The spectral function of an elliptic operator. Acta Math. 121 (1968), 193218. MR 0609014 Zbl 0164.13201

[20] A. A. Ilyin, Lower bounds for the spectrum of the Laplace and Stokes operators. Discrete Contin. Dyn. Syst. 28 (2010), no. 1, 131-146. MR 2629475 Zbl 1190.35171

[21] V. Y. Ivrii, On the second term of the spectral asymptotics for the Laplace-Beltrami operator on manifolds with boundary. Funtsional. Anal. i Prilozhen. 14 (1980), no. 2 , 25-34. MR 0575202 Zbl 0448.58024

[22] V. Y. Ivrii, Microlocal analysis and precise spectral asymptotics. Springer Monographs in Mathematics, Springer-Verlag, Berlin, 1998. MR 1631419 Zbl 0906.35003

[23] H. Kovařík, S. Vugalter, and T. Weidl, Two dimensional Berezin-Li-Yau inequalities with a correction term. Comm. Math. Phys. 287 (2009), no. 3, 959-981. MR 2486669 Zbl 0906.35003

[24] M. L. Lapidus, Fractal drum, inverse spectral problems for elliptic operators and a partial resolution of the Weyl-Berry conjecture. Trans. Amer. Math. Soc. 325 (1991), no. 2, 465-529. MR 0994168 Zbl 0741.35048

[25] A. Laptev, Dirichlet and Neumann eigenvalue problems on domains in Euclidean spaces. J. Funct. Anal. 151 (1997), no. 2, 531-545. MR 1491551 Zbl 0892.35115

[26] A. Laptev and T. Weidl, Sharp Lieb-Thirring inequalities in high dimensions. Acta Math. 184 (2000), no. 1, 87-111. MR 1756570 Zbl 1142.35531

[27] P. Li and S. T. Yau, On the Schrödinger equation and the eigenvalue problem. Comm. Math. Phys. 88 (1983), no. 3, 309-318. MR 0701919 Zbl 0554.35029

[28] R. B. Melrose, Weyl's conjecture for manifolds with concave boundary, Geometry of the Laplace operator (Proc. Sympos. Pure Math., Univ. Hawaii, Honolulu, Hawaii, 1979). Proc. Sympos. Pure Math., XXXVI, Amer. Math. Soc., Providence, R.I., 1980, pp. 257274. MR 0573438 Zbl 0436.58024 
[29] A.D. Melás, A lower bound for sums of eigenvalues of the Laplacian. Proc. Amer. Math. Soc. 131 (2003), 631-636. MR 1933356 Zbl 1015.58011

[30] R. Osserman, A note on Hayman's theorem on the bass note of a drum, Comment. Math. Helv. 52 (1977), no. 4, 545-555. MR 0459099 Zbl 0374.52008

[31] Y. Safarov and D. Vassiliev, The asymptotic distribution of eigenvalues of partial differential operators. Transl. of Math. Monog. 155, Amer. Math. Soc., Providence, RI, 1997. MR 1414899 Zbl 0870.35003

[32] M. van den Berg, A uniform bound on trace $\left(e^{t \Delta}\right)$ for convex regions in $\mathbb{R}^{n}$ with smooth boundaries. Comm. Math. Phys. 92 (1984), no. 4, 525-530. MR 0736409

[33] T. Weidl, Improved Berezin-Li-Yau inequalities with a remainder term. Amer. Math. Soc. Transl. 225 (2008), no. 2, 253-263. MR 2509788 Zbl 1168.35404

[34] H. Weyl, Das asymptotische Verteilungsgesetz der Eigenwerte linearer partieller Differentialgleichungen (mit einer Anwendung auf die Theorie der Hohlraumstrahlung). Math . Ann. 71 (1912), no. 4, 441-479. MR 1511670 JFM 43.0436.01

[35] I. M. Yaglom and V. G. Boltyanski, Convex figures. Holt, Reinahrt and Winston, New York, 1961. Zbl 0098.35501

[36] S. Y. Yolcu, An improvement to a Berezin-Li-Yau type inequatliy. Proc. Amer. Math. Soc. 138 (2010), 4059-4066. MR 2679626 Zbl 1202.35366

[37] S. Y. Yolcu and T. Yolcu, A Berezin-Li-Yau type inequality for the fractional Laplacian on a bounded domain. Preprint 2010. arXiv:1009.4270v1

Received October 23, 2010, revised November 19, 2010

Leander Geisinger, Universität Stuttgart, Pfaffenwaldring 57, 70569 Stuttgart, Germany

E-mail: geisinger@mathematik.uni-stuttgart.de

Ari Laptev, Imperial College London, 180 Queen's Gate, London SW7 2AZ, UK

E-mail: a.laptev@imperial.ac.uk

Timo Weidl, Universität Stuttgart, Pfaffenwaldring 57, 70569 Stuttgart, Germany

E-mail: weidl@mathematik.uni-stuttgart.de 